\title{
Hyperglycemic hyperosmolar nonketotic syndrome with rhabdomyolysis in a patient with newly-onset diabetes: one case report and literature review
}

\author{
Xin Hu ${ }^{2}$, Juan Wang ${ }^{1}$, Shuang Liang ${ }^{3}$, Yueting Zhao ${ }^{2}$, Shuhang Xü ${ }^{2 *}$ and Chao Liu ${ }^{2}$ \\ ${ }^{1}$ Jiangning Hospital of Traditional Chinese Medicine, Nanjing, China \\ ${ }^{2}$ Endocrine and Diabetes Center, Affiliated Hospital of Integrated Traditional Chinese and Western Medicine, Nanjing University of Chinese Medicine, Jiangsu \\ Province Academy of Traditional Chinese Medicine, Nanjing, China \\ ${ }^{3}$ Rui'en Diabetes Hospital, Chengdu, China
}

\section{Introduction}

Hyperglycemic hyperosmolar nonketotic syndrome (HHNS) is one of the most severe acute complications of diabetes. The hallmark of HHNS is profound dehydration, marked hyperglycemia, and often some degree of neurologic impairment with mild or no ketosis [1]. Rhabdomyolysis is an unusual but underestimated complication of HHNS [2]. HHNS accompanied with rhabdomyolysis in diabetic patients was reported 20 years ago [3]. The prognosis of HHS is worse. Herein, we report one patient newly diagnosed as type 2 diabetes mellitus and complicated with HHNS and rhabdomyolysis. The relevant literatures are reviewed to improve the cognition of the diseases.

\section{Case Report}

A 56-year-old female had complained of poor appetite and fatigue for 2 weeks. She was healthy previously. She went to another hospital 14 days ago. The antibiotic solution has been given intravenously for 3 days. But her symptoms became aggravated gradually with polydipsia and polyuria, and then she was sent to emergency room in our hospital. The laboratory inspection showed the leukocytes, neutrophils, C-reacting protein (CRP), blood glucose, serum sodium, and serum myocardial enzymes levels were extremely high. Urine glucose was positive; however, urine ketones were negative. The arterial blood gas analysis didn't indicate obvious acidosis. The chest CT revealed pulmonary infection.

When admitted, her body temperature was $36.7^{\circ} \mathrm{C}$, heart rate was 82 beats/minutes, respiratory rate was $18 / \mathrm{min}$, and blood pressure was $124 / 64 \mathrm{mmHg}$. Her consciousness was clear with flagging mind. The whole body skin was dry, and her eye sockets didn't appear to be sunken. The breath sounds were rough. The myodynamia of two legs declined. The Laboratory examinations revealed leukocytes $27.72 \times 10^{9} / \mathrm{L}$; serum glucose $38.86 \mathrm{mmol} / \mathrm{L}$; sodium $165.5 \mathrm{mmol} / \mathrm{L}$; chloride $116.4 \mathrm{mmol} / \mathrm{L}$; potassium $3.35 \mathrm{mmol} / \mathrm{L}$; albumin $31 \mathrm{~g} / \mathrm{L}$; serum urea $15.5 \mathrm{mmol} / \mathrm{L}$; creatinine $83 \mathrm{umol} / \mathrm{L}$; and $\mathrm{cTnT} 11.62 \mathrm{pg} / \mathrm{ml}$. The calculated serum osmolality came to $392.1 \mathrm{mOsm} / \mathrm{L}$. Because her urine color was found to be dark red, the laboratory evaluation of lactate dehydrogenase $(\mathrm{LDH})$, creatine kinase $(\mathrm{CK})$, creatine kinase-MB (CK-MB), and myoglobin was performed. The results revealed LDH 1076U/L, CK 686U/L, CK-MB 28U/L, and myoglobin $>500 \mathrm{ng} / \mathrm{mL}$. She was diagnosed with dabetes, HHNS, rhabdomyolysis, pulmonary infection (Table 1).

The patient was immediately treated with fluid resuscitation and insulin infusion. Parallelly, antibiotics, proton-pump inhibitor, potassium, etc. was given as a routine. To prevent acute renal failure, hydration was accomplished by intravenous and oral fluid therapy with isotonic fluids at a rate that maintained a urinary flow of $200 \mathrm{~mL} / \mathrm{h}$ until CK levels decreased. After about $8000 \mathrm{ml}$ of fluid was administrated intravenously, the patient was found to have chemosis and lower limb edema. Therefore, 2000-3000ml extra water was supplied every day by nasal feeding. After 2 days continuous hydration and insulin treatment, the blood glucose, serum sodium, and osmolality decreased significantly, and insulin pump was given to achieve a better blood glucose control. However, levels of creatine, creatine kinase-MB (CK$\mathrm{MB}$ ), and myoglobin increased. At day 4 after admission, her blood glucose and sodium kept stable, and CK decreased significantly. The patient's urine changed to be clear. Her consciousness was restored; however, her muscle strength had not improved. After fluid resuscitation and continuous subcutaneous insulin infusion, the patient's blood sugar, sodium, CK, CK-MB, and myoglobulin levels gradually decreased. After 9 days treatment, the levels of CK, CK-MB, and myoglobulin were only $632 \mathrm{U} / \mathrm{L}, 20 \mathrm{U} / \mathrm{L}$ and $294 \mathrm{ng} / \mathrm{ml}$, respectively. The patient's muscle strength was significantly improved, and could walk.Her appetite was remarkably increased. On the 14th day of hospitalization, the continuous examination revealed that her $\mathrm{CK}, \mathrm{CK}-\mathrm{MB}$, and myoglobulin levels were nearly normal. Then the insulin pump was switched to multiple daily injections. After the blood glucose was well controlled, she was discharged.

\section{Discussion}

HHNS is characterized by severe hyperglycemia $(>33 \mathrm{mmol} / \mathrm{l}$ or $600 \mathrm{mg} / \mathrm{dl})$ with marked serum hyperosmolarity $(>320 \mathrm{mmol} / \mathrm{kg})$ and without evidence of significant ketosis [4]. The typical patients are older and have undiagnosed diabetes or type 2 diabetes managed by

Correspondence to: Shuhang $\mathrm{Xu}$, Endocrine and Diabetes Center, Affiliated Hospital of Integrated Traditional Chinese and Western Medicine, Nanjing University of Traditional Chinese Medicine, Jiangsu Province Academy of Traditional Chinese Medicine, No.100 Shizi Street, Hongshan Road, 210028, Nanjing, China, Tel: 86-25-8560 8778; E-mail: shuhangxu@163.com

Chao Liu, Endocrine and Diabetes Center, Affiliated Hospital of Integrated Traditional Chinese and Western Medicine, Nanjing University of Traditional Chinese Medicine; Jiangsu Province Academy of Traditional Chinese Medicine No.100, Hongshan Road, 210028, Nanjing, China, E-mail: liuchao@nfmcn.com

Received: June 15, 2017; Accepted: June 23, 2017; Published: June 30, 2017 
Table 1. Laboratory findings before and after admission.

\begin{tabular}{|c|c|c|c|c|c|c|c|c|}
\hline \multirow{2}{*}{ Laboratory tests } & \multicolumn{8}{|c|}{ Days after admission } \\
\hline & 1 & 2 & 3 & 4 & 6 & 9 & 12 & 14 \\
\hline Lecukocyte $\left(\times 10^{9} / \mathrm{L}\right)$ & 27.72 & 22.07 & 20.55 & 17.63 & 6.23 & 10.55 & 5.87 & 5.11 \\
\hline Glucose $(\mathrm{mmol} / \mathrm{L})$ & 38.86 & 13.24 & 12.47 & 8.93 & 6.69 & 4.19 & 6.41 & 6.2 \\
\hline Sodium $(\mathrm{mmol} / \mathrm{L})$ & 165.5 & 174.8 & 144.1 & 150.2 & 145.7 & 147.3 & 140.6 & 142 \\
\hline Potassium $(\mathrm{mmol} / \mathrm{L})$ & 3.35 & 3.29 & 3.82 & 3.39 & 3.37 & 3.8 & 3.88 & 3.64 \\
\hline CK (U/L) & 686 & 15030 & 23600 & 15520 & 8090 & 632 & 75 & 48 \\
\hline CK-MB (U/L) & 28 & 188 & 170 & 249 & 174 & 20 & 7 & 9 \\
\hline Myoglobin (ng/mL) & $>500$ & $>500$ & $>500$ & $>500$ & $>500$ & 294 & 122 & 73.5 \\
\hline Plasma osmolality & 376.56 & 369.42 & 308.31 & 316.11 & 304.83 & 306.39 & 295.37 & 297.48 \\
\hline $24 \mathrm{~h}$ input & 10h:3520 & 6085 & 4535 & 6272 & 3935 & - & 2000 & 2000 \\
\hline 24 h output & $10 \mathrm{~h}: 2300$ & 3300 & 5500 & 3000 & 5000 & - & 1600 & 2000 \\
\hline
\end{tabular}

diet and/or oral diabetic medication. It is worth noting that insulin is still present in these patients, probably explained by the residual insulin secretory capacity retained by diabetes. The insulin level remains sufficient to suppress lipolysis and to avoid significant keto acid production; however, it is not adequate to reduce blood glucose levels, particularly in the presence of significant insulin resistance. Therefore, marked hyperglycemia $(>33 \mathrm{mmol} / \mathrm{l}$ or $600 \mathrm{mg} / \mathrm{dl})$ in the absence of ketoacidosis is the usual finding. The concurrent acid-base disturbance is commonly absent, and arterial $\mathrm{pH}$ rarely drops below 7.30. Based on these characteristics, the patient was diagnosed with type 2 diabetes and HHNS.

The patient had dark urine and muscle weakness, and her plasma CK, CK-MB, myoglobin levels were remarkably elevated, the troponin was normal, and the patient didn't have any manifestations of myocardial infarction, and the electrocardiogram showed only tachycardia. Since the clinical evidences of myonecrosis and release into the systemic circulation of muscle cell contents, including myoglobin, creatinine, $\mathrm{CK}$, the diagnosis of rhabdomyolysis was made. It was reported that more than $50 \%$ of the HHNS patients are companied with rhabdomyolysis[3]. Its severity ranges from mild elevated CK level with no symptoms to significant elevated CK level with acute renal failure that may require hemodialysis[2].

Rhabdomyolysis is a complex and rare medical condition which involves the rapid breakdown of damaged or injured skeletal muscle. Its characteristic is the fracture and necrosis of muscle fibers, leading to the release of cell degradation products and intracellular elements within the bloodstream and extracellular space. Notably, the percentage of patients with rhabdomyolysis who develop acute kidney injury, the most dramatic consequence, varies from $13 \%$ to over $50 \%$ according to both the cause and the clinical and organizational setting where they are diagnosed [5,6]. Direct muscle injury is considered as the most common cause of rhabdomyolysis; besides, there are other causes such as hereditary enzyme disorders, drugs, toxins, endocrinopathies, malignant hyperthermia, neuroleptic malignant syndrome, heatstroke, hypothermia, electrolyte alterations, diabetic ketoacidosis and nonketotic hyperosmolar coma, severe hypo/hyperthyroidism and bacterial or viral infections [7].

The mechanism of HHNS resulting in rhabdomyolysis is still not specific. The elevated blood glucose, sodium and urea in HHNS patients resulted in increase of the plasma osmolality, causing serious dehydration, hypovolemia and oxygen deficiency of local tissue $[8,9]$. As a consequence, the $\mathrm{Na} / \mathrm{K}$ ATPase pump of skeletal muscle is destroyed, and the osmotic pressure imbalance inside and outside the skeletal muscle cells ultimately leads to the damage and dissolve of muscle cells. A pathological interaction between actin and myosin and activation of cell protease was triggered, with subsequent necrosis of muscle fibers, release of potassium, phosphates, myoglobin, CK and urates into the extracellular space and into the bloodstream. Myoglobin can precipitate in the glomerular filtrate, particularly in an acidic environment, causing tubular occlusion and severe kidney damage [5].

HHNS is a severe metabolic disorder of diabetes, and the occurrence of rhabdomyolysis increase the severity. Acute renal failure is the most serious consequence of rhabdomyolysis, and occurs in up to $67 \%$ of all cases. During the treatment, adequate hydration, effective control of blood glucose and electrolytes, and frequent monitoring throughout the first few days of admission may be helpful to reduce the severity and incidence of rhabdomyolysis. Once rhabdomyolysis is suspected, a rapid and larger fluid replacement should be continued with the goal of maintaining a urinary flow of $200 \mathrm{ml} / \mathrm{h}$. On the other hand, it is important to monitor diuresis rate and stop fluid infusion if signals of overhydration appear. To avoid volume overload, an alternate $500 \mathrm{ml}$ of sterile saline solution with $500 \mathrm{ml}$ of $5 \%$ glucose solution is recommended, adding $50 \mathrm{mmol}$ of sodium bicarbonate for each subsequent 2-3 liter of solution (usually 20-300 mmol on the first day). The goal is to maintain the urine $\mathrm{pH}$ above 6.5 and plasma $\mathrm{pH}$ below 7.50 [5].If refractory hypercalcemia, metabolic acidosis, or refractory oligoanuria is present, renal replacement therapy is required [10].

In the end, we reported one case of patient diagnosed with rhabdomyolysis caused by HHNS and type 2 diabetesin China. The HHNS complicated with rhabdomyolysis was found when diabetes was firstly documented. Early recognition of rhabdomyolysis complicated with HHNS Rapid, adequate fluid resuscitation and intensive monitoring are crucial to prevent acute renal failure and reduce the mortality.

\section{Conflict of Interest}

The authors declare that there are no any financial or personal relationships with other people or organizations that could inappropriately influence this work.

\section{Acknowledgement}

The authors gratefully acknowledge the grand support of National Natural Science Foundation of China (Grant number: 81200577) and "Six Talent Peak" Project of Jiangsu Province under Grant (NO 2013WSN-063).

\section{References}

1. Stoner GD (2005) Hyperosmolar hyperglycemic state. Am Fam Physician 71: 1723-1730.

2. Gangopadhyay KK, Ryder RE (2006) Nontraumatic rhabdomyolysis: an unusual complication of diabetic hyperosmolar nonketotic (HONK) state. J R Soc Med 99: 200. [Crossref]

3. Singhal PC, Abramovici M, Venkatesan J (1990) Rhabdomyolysis in the hyperosmolal state. Am J Med 88: 9-12.[Crossref] 
Hu X (2017) Hyperglycemic hyperosmolar nonketotic syndrome with rhabdomyolysis in a patient with newly-onset diabetes: one case report and literature review

4. McCombs DG, Appel SJ, Ward ME (2015) Expedited diagnosis and management of inpatient hyperosmolar hyperglycemic nonketotic syndrome. $J$ Am Assoc Nurse Pract 27: 426-432. [Crossref]

5. Cervellin G, Comelli I, Lippi G (2010) Rhabdomyolysis: historical background, clinical, diagnostic and therapeutic features. Clin Chem Lab Med 48: 749-756. [Crossref]

6. Torres PA, Helmstetter JA, Kaye AM, Kaye AD (2015) Rhabdomyolysis: pathogenesis, diagnosis, and treatment. Ochsner J 15: 58-69. [Crossref]

7. Bosch X, Poch E, Grau JM (2009) Rhabdomyolysis and acute kidney injury. N Engl JMed 361: 62-72.
8. Izumi T, Shimizu E, Imakiire T, Kikuchi Y, Oshima S, et al. (2010) A successfully treated case of hyperosmolar hyperglycemic state complicated with rhabdomyolysis, acute kidney injury, and ischemic colitis. Intern Med 49: 2321-2326. [Crossref]

9. Keltz E, Khan FY, Mann G (2013) Rhabdomyolysis. The role of diagnostic and prognostic factors. Muscles Ligaments Tendons J 3: 303-312. [Crossref]

10. Panizo N, Rubio-Navarro A, Amaro-Villalobos JM, Egido J, Moreno JA (2015) Molecular Mechanisms and Novel Therapeutic Approaches to RhabdomyolysisInduced Acute Kidney Injury. Kidney Blood Press Res 40: 520-532. [Crossref]

Copyright: (C2017 Hu X. This is an open-access article distributed under the terms of the Creative Commons Attribution License, which permits unrestricted use, distribution, and reproduction in any medium, provided the original author and source are credited. 